$\mathbb{T}$ periodica polytechnica

Chemical Engineering

$53 / 1(2009) 9$

doi: 10.3311/pp.ch.2009-1.02

web: http://www.pp.bme.hu/ch

(c) Periodica Polytechnica 2009

RESEARCH ARTICLE

\section{A study on the equilibrium reaction of benzaldehyde and sodium bisulphite by in situ Fourier transform IR spectroscopy}

Péter Sallay / Nikolett Szilágyi / István Csontos / György Keglevich

Received 2009-04-23

\begin{abstract}
The addition of sodium bisulphite to benzaldehyde was monitored at different conditions by in situ Fourier transform IR spectroscopy and the equilibrium constant was determined.
\end{abstract}

\section{Keywords}

in situ Fourier transform IR spectroscopy · monitoring · equilibrium · benzaldehyde - sodium bisulphite adduct

\section{Acknowledgement}

The authors are grateful for the support from the Hungarian Scientific Research Found (OTKA, T067679).

\section{Péter Sallay}

Nikolett Szilágyi

István Csontos

\section{György Keglevich}

Department of Organic Chemistry and Technology, BME, Budafoki út 8. H1111 Budapest, Hungary

\section{Introduction}

In situ Fourier transform IR spectroscopy is a suitable method for monitoring a variety of organic chemical reactions [1]-[8]. The time-dependent IR spectra reveal the concentration profile of the components, which allows formal kinetic measurements, determination of equilibrium constants [2], and optimization of the reaction studied. This latter is important from the point of view of environmentally friendly chemistry. It is also possible to identify intermediates [7,8] in the mixture and hence evaluate reaction mechanisms. In situ Fourier transform IR spectroscopy is a suitable tool to study organic chemical reactions over a wide range of temperatures, and even under pressure. The spectrometer is supplied with an attenuated total reflection (ATR) probe that is in contact with the reaction mixture. In situ Fourier transform IR has become a routine method in the fine chemical, pharmaceutical, and even plastics industries. Heterogeneous phase reactions can also be monitored by in situ Fourier transform IR spectroscopy [8].

\section{Experimental part}

2.1 Materials and methodology

The ReactIR apparatus used in the experiments was of Labmax, Mettler Toledo Internat. Inc. type.

The compounds used were of commercial quality $\left(\mathrm{Na}_{2} \mathrm{~S}_{2} \mathrm{O}_{5}\right.$, Reanal, benzaldehyde, Sigma Aldrich).

The easily decomposable benzaldehyde-sodium bisulphite adduct was freshly prepared for IR examination according to the following:

At room temperature, $14.6 \mathrm{~g}$ of pyrosulphite $\left(\mathrm{Na}_{2} \mathrm{~S}_{2} \mathrm{O}_{5}\right)$ (equal to $0.153 \mathrm{~mol}$ of $\mathrm{NaHSO}_{3}$ ) was dissolved in $75 \mathrm{ml}$ of water, and $10.5 \mathrm{ml}(0.104 \mathrm{~mol})$ of benzaldehyde was added. The immediately separated whitish precipitate was filtered, dried in a desiccator, and its IR spectrum was taken in $\mathrm{KBr}$ pellet.

The ReactIR experiments were carried out according to the following:

We dissolved $4.9 \mathrm{~g}$ of sodium pyrosulphite (equivalent to $0.051 \mathrm{~mol}$ of $\mathrm{NaHSO}_{3}$ ) in $35 \mathrm{ml}$ of distilled water, and then we added in three steps the benzaldehyde, in the quantities given

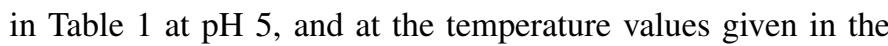


Table 1 Until the third addition of the benzaldehyde, the reaction mixture remained homogeneous. We have continuously monitored the reactions through the ReactIR equipment.

Tab. 1. Experimental data

\begin{tabular}{lccc}
\hline Reaction & $\begin{array}{c}\text { Bisulphite: } \\
\text { benzaldehyde } \\
\text { molar ratio }\end{array}$ & $\begin{array}{c}\text { Quantity of } \\
\text { benzaldehyde } \\
(\mathrm{ml})\end{array}$ & $\begin{array}{c}\text { Temperature } \\
\left({ }^{\circ} \mathrm{C}\right)\end{array}$ \\
\hline Exp. 1/1. step & 3.5 & 1.5 & 26 \\
Exp. 1/2. step & 2.1 & 1.0 & 26 \\
Exp. 1/3. step & 1.5 & 1.0 & 26 \\
Exp. 2 & 1.5 & 3.5 & 60 \\
\hline
\end{tabular}

\section{Results and discussion}

The reaction examined is the addition of $\mathrm{NaHSO}_{3}$ to benzaldehyde. The bisulphite has been prepared in situ, through the reaction of pyrosulphite and water (reactions 1 and 2):

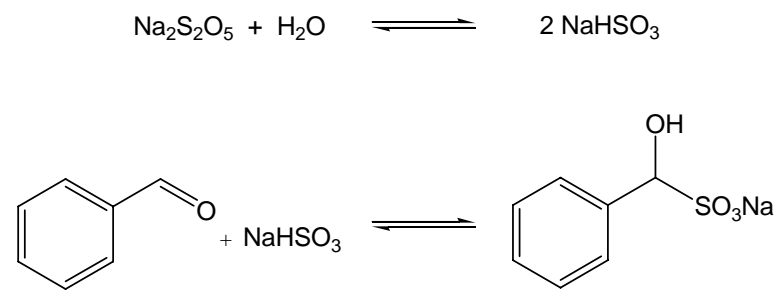

At first, we have taken the IR spectra of the starting materials, as well as that of the bisulphite-benzaldehyde adduct. Some characteristic absorptions of the components are shown in Table 2 .

Tab. 2. Characteristic IR absorptions of the reaction components in the range of $900-1300 \mathrm{~cm}^{-1}$

\begin{tabular}{|c|c|c|c|c|}
\hline \multirow[t]{2}{*}{ Benzaldehyde $^{a}$} & \multirow[t]{2}{*}{$\mathrm{NaHSO}_{3}^{a}$} & \multirow[t]{2}{*}{$\mathrm{Na}_{2} \mathrm{~S}_{2} \mathrm{O}_{5}^{b}$} & \multicolumn{2}{|c|}{$\begin{array}{l}\text { Bisulphite-benzaldehyde } \\
\text { adduct }\end{array}$} \\
\hline & & & a & $b$ \\
\hline & 965 & 978 & & \\
\hline & 1022 & & 1034 & 1035 \\
\hline & $1053^{c}$ & 1057 & & \\
\hline \multirow[t]{2}{*}{1070} & & 1066 & $1069^{d}$ & 1070 \\
\hline & & & $1089^{d}$ & 1088 \\
\hline \multirow[t]{2}{*}{1165} & $1165^{e}$ & & & \\
\hline & & 1184 & 1182 & 1190 \\
\hline $1204^{f}$ & $1204^{e}$ & & 1204 & 1215 \\
\hline
\end{tabular}

${ }^{a}$ ReactIR, solution in water; ${ }^{b}$ Bruker Tensor 37 in $\mathrm{KBr}$ pellet; ${ }^{c} \mathrm{SO}_{3}$ symmetric stretching vibration; ${ }^{d} \mathrm{C}-\mathrm{O}$ stretching vibration; ${ }^{e} \mathrm{SO}_{3}$ asymmetric stretching vibration; $f$ ring $\mathrm{C}-\mathrm{CHO}$ stretching vibration.

From the data in Table 2 one can see that, the ReactIR is suitable for the separate detection of the reaction components. This can be seen on the 3D spectrum shown in Fig. 1, which represents the steps of Experiment 1. In the figure one can see the rapid adduct formation, as well as the reduction of the quantity of sodium bisulphite.
Deconvolution diagrams of the reactions give only relative concentration values (Fig. 2).

Determination of the equilibrium constants requires the conversion of the relative concentration values to real concentrations. To this aim we had to check the validity of the LambertBeer law. The relative concentrations can be treated as pseudoabsorbances in the calculations. Fig. 2 shows that, the relative concentrations of both the bisulphite and the bisulphitebenzaldehyde adduct are proportional to the quantity of the corresponding substance (the corresponding value of absorbance and the amount of benzaldehyde added are constant), so the Lambert-Beer law is valid even in the rather concentrated solution used by us. We have checked the validity of this law also in the case of the solution of sodium pyrosulphite. The correlation was approximately linear $\left(\mathrm{R}^{2}=0.9723\right)$, but found a better, polynomial $\left(\mathrm{R}^{2}=0.999976\right)$ form.

The equilibrium concentration of bisulphite can be calculated by means of the Eq. 3.a. but we used a more accurate, empirical Eq. 3.b

$$
\begin{aligned}
C_{\mathrm{NaHSO}_{3}}^{\infty}= & \frac{C_{\mathrm{NaHSO}_{3}}^{0} \cdot A_{\mathrm{NaHSO}_{3}}^{\infty}}{A_{\mathrm{NaHSO}_{3}}^{0}} \\
C_{\mathrm{NaHSO}_{3}}^{\infty}= & 0.03463 \cdot\left(A_{\mathrm{NaHSO}_{3}}^{\infty}\right)^{3}+ \\
& 0.07565 \cdot\left(A_{\mathrm{NaHSO}_{3}}^{\infty}\right)^{2}+0.4430 \cdot\left(A_{\mathrm{NaHSO}_{3}}^{\infty}\right)
\end{aligned}
$$

where: $\quad \mathrm{C}=$ bisulphite concentration ( $\mathrm{mol} / \mathrm{l})$

$\mathrm{A}=$ pseudo-absorbance

$0=$ initial status

$\infty=$ state of equilibrium

The concentration of the adduct is equal to the difference between the initial and equilibrium bisulphite - concentration (Eq. 4):

$$
C_{\text {adduct }}^{\infty}=C_{\mathrm{NaHSO}_{3}}^{0}-C_{\mathrm{NaHSO}_{3}}^{\infty}
$$

In the calculation of the initial bisulphite concentration, we have also taken into account equilibrium between pyrosulphite and bisulphite. The equilibrium constant at 26 and $60^{\circ} \mathrm{C}\left(\mathrm{K}_{26}=22.8\right.$ $1 / \mathrm{mol}, \mathrm{K}_{60}=46.9 \mathrm{l} / \mathrm{mol}$ ) was taken from the literature [9].

The equilibrium constant for the reaction studied was calculated by means of Eq. 5 .

$$
K_{C}=\frac{C_{\text {adduct }}^{\infty}}{C_{\mathrm{NaHSO}_{3}}^{\infty} \cdot\left(C_{\text {benzaldehyde }}^{0}-C_{\text {adduct }}^{\infty}\right)}
$$

After substituting the respective values in the formula, the following equilibrium constants were obtained for the case of different initial molar ratios and temperatures (Table 3):

The values of the equilibrium constants measured by us are smaller than those reported in the literature $\left(0.44-11.3 \cdot 10^{3}\right.$ [10]). However, it is known that [10], the ionic strength exerts significant influence on the value of the equilibrium constant: when the ionic strength increases ten times (from $0.1 \mathrm{~mol} / \mathrm{l}$ to $1.0)$, the value of the equilibrium constant will decrease from $4.81 \mathrm{l} / \mathrm{mol}$ to $0.98 \mathrm{l} / \mathrm{mol}$. As we have performed our measuring 
Fig. 1. Reaction of benzaldehyde and sodium bisulphite (Table 1 reaction 1/steps 1 and 2).
Fig. 2. Changes in the relative concentrations of bisulphite, benzaldehyde and the bisulphitebenzaldehyde adduct depending on time after addition of benzaldehyde portions.
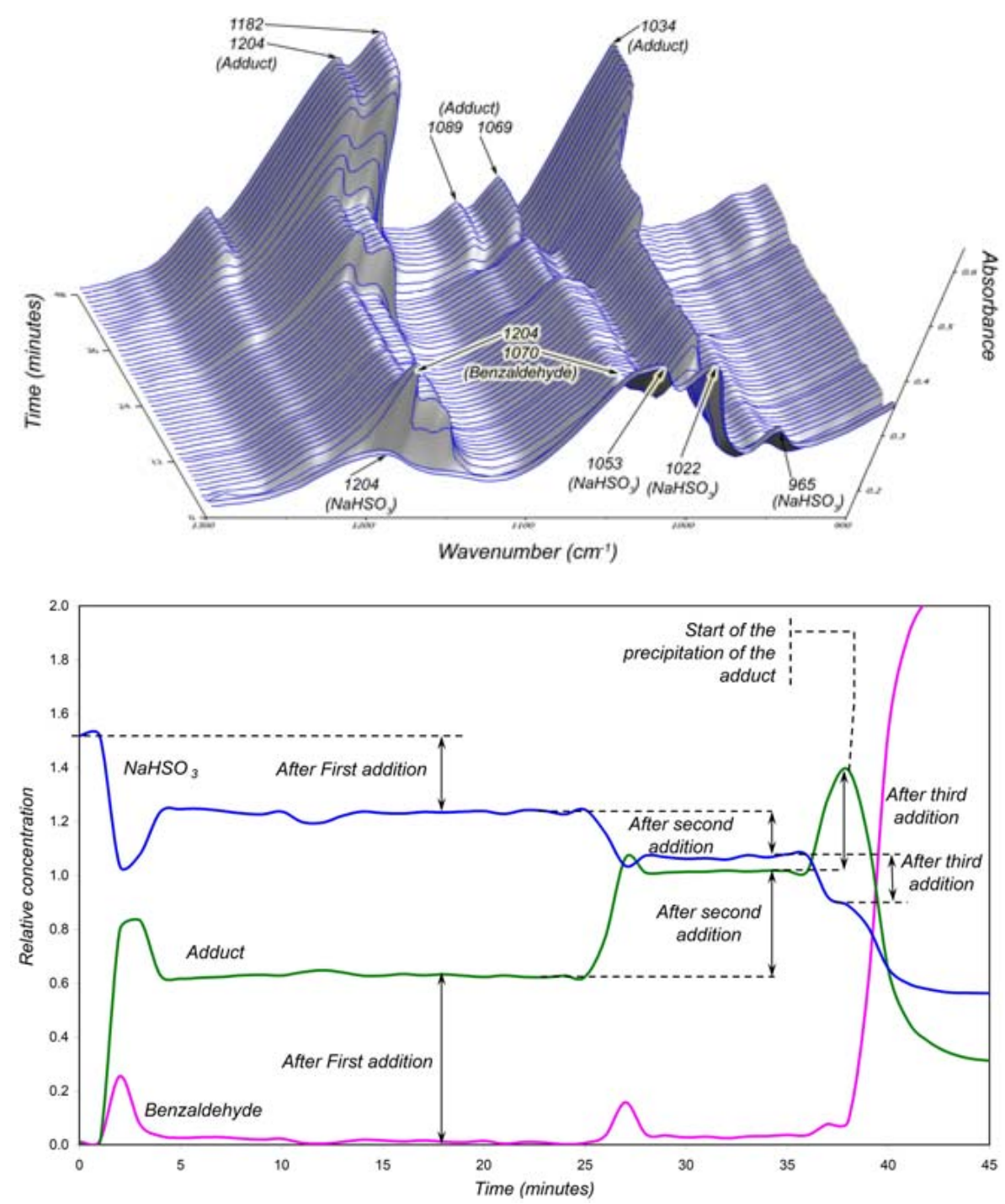

Tab. 3. Equilibrium constants for the reaction of benzaldehyde and sodium bisulphite

\begin{tabular}{ccc}
\hline $\begin{array}{c}\text { Bisulphite:benzaldehyde } \\
\text { molar ratio }\end{array}$ & $\begin{array}{c}\text { Temperature } \\
\left({ }^{\circ} \mathrm{C}\right)\end{array}$ & $\begin{array}{c}\text { Equilibrium constant } \\
(\mathrm{I} / \mathrm{mol})\end{array}$ \\
\hline 3.5 & 26 & 5.215 \\
2.1 & 26 & 5.235 \\
1.5 & 26 & 5.502 \\
1.5 & 60 & 8.484 \\
\hline
\end{tabular}

at higher concentrations than usual, we have calculated also the ionic strength (I) applied during our experiments:

$$
I=\frac{1}{2} \sum_{B=1}^{n} c_{B} z_{B}^{2}
$$

where: $c_{B}$ is the concentration (mol/l) of ion $B$ and $z_{B}$ is the charge-number of ion $\mathrm{B}$.

In our case the ion-intensity was $1.48 \mathrm{~mol} / \mathrm{l}$, so presumably the smaller equilibrium constants measured by us was a consequence of the higher concentrations, that is of the higher ionic strength.

We have disregarded the dependence of the equilibrium con- stant between pyrosulphite from the ionic strength, as the equilibrium is entirely shifted to the right side of the equation.

By means of the van t'Hoff equation, one can also calculate the reaction heat from the various equilibrium constants (Table 3 ) measured at different temperatures $\left(26\right.$ and $\left.60{ }^{\circ} \mathrm{C}\right)$. Applying a 1.5:1 bisulphite-benzaldehyde ratio this is $-2.52 \mathrm{~kJ} / \mathrm{mol}$, that is, the temperature of the reaction does not exert any significant influence on equilibrium of the reaction.

Therefore we came to the conclusion that, the ReactIR apparatus can successfully be applied for the monitoring of the equilibrium reactions and for the determination of the equilibrium constant.

\section{Summary}

In our work we have studied a well-known reaction, the equilibrium addition reaction between benzaldehyde and sodium bisulphite. The reaction was followed by in situ Fourier transform IR spectroscopy. We have determined the equilibrium constant of the rapidly achieved equilibrium at different initial molar ratios and different temperatures. The values of the equilibrium constant proved to be smaller than those given by the literature. We demonstrated that, this was a consequence of the higher con- 
centrations, that is, higher ionic strength, as we have performed our measurements at higher concentrations than usual.

\section{References}

1 Ende D J, Preigh M J, Process optimization with in situ technologies, Current Opinion in Drug Discovery \& Development 3 (2000), 699-706.

2 Keglevich G, Csontos I, Novák T, Mucsi Z, Marosi G, Greiner I;, Monitoring of esterifications by in situ Fourier transformation spectroscopy, Magyar Kémiai Folyóirat 112 (2006), 114-116.

3 Pintar A, Batista J, Levec J, In situ Fourier transform infrared spectroscopy as an efficient tool for determination of reaction kinetics, Analyst 127 (2002), 1535-1540, DOI 10.1039/b207204a.

4 Rein A J, SPIE - Optically Based Methods for Process Analysis, Vol. 1681, 1992.

5 Sheridan R E, Rein A J, FTIR system reveals secrets of chemical processes, R\&D Magazine 33 (1991), 100-102.

6 Yamagiwa N, Abiko Y, Sugita M, Tian J, Matsunaga S, Shibasaki $\mathbf{M}$, Catalytic asymmetric cyano-phosphorylation of aldehydes using a YLi(3)tris(binaphthoxide) complex (YLB), Tetrahedron: Asymmetry 17 (2006), 566-573.

7 Keglevich G, Csontos I, Szilágyi N, Greiner I, A study of the $\mathrm{pH}$ dependence of the two-step oximation of acetone by in situ Fourier transform infrared spectroscopy, Chemical Engineering Technology 31 (2008), 421-425, DOI 10.1002/ceat.200700448.

8 Keglevich G, Csontos I, Szilágyi N, Monitoring the pH-dependent oximation of methyl ethyl ketone and benzaldehyde by in situ Fourier transform IR spectroscopy in a heterogeneous liquid - liquid two-phase system, Spectroscopy Letters 42 (2009), 67-72, DOI 10.1080/00387010802428476.

9 Ermatchkov V, Kamps A P-S, Maurer G, The chemical reaction equilibrium constant and standard molar enthalpy change for the reaction 2 $\mathrm{NaHSO}_{3}$ - Na2S2O5 + H2O: a spectroscopic and calorimetric investigation, Journal of Chemical Thermodynamics 37 (2005), 187-199, DOI 10.1016/j.jct.2004.08.011.

10 Olson T M, Boyce S D, Hoffmann M R, Kinetics, thermodynamics, and mechanism of the formation of benzaldehyde-S(IV) adducts, Journal of Physical Chemistry 90 (1986), 2482-2488, DOI 10.1021/j100402a043. 